\title{
ACTIVIDAD SÍSMICA PERCIBIDA EN COSTA RICA DURANTE EL 2017
}

\author{
FELT SEISMICITY IN COSTA RICA DURING 2017
}

\author{
Juan L. Porras*, Lepolt Linkimer, Ivonne G. Arroyo, Mario Arroyo \\ y Magda Taylor
}

\author{
Red Sismológica Nacional (RSN: UCR-ICE), Apdo. 214-2060, \\ San Pedro, Costa Rica \\ * Autor para contacto: juan.porrasloria@ucr.ac.cr
}

\begin{abstract}
During 2017, the National Seismological Network (RSN: UCR-ICE) located 2755 earthquakes and reported 216 felt events, including four significant earthquakes with magnitudes Mw between 5.5 and 6.3. The majority $(91 \%)$ of the felt earthquakes were shallow $(<30 \mathrm{~km})$ and $63 \%$ had low magnitudes $(\mathrm{Mw}<3.9)$. Local and regional faulting caused $62 \%$ of the felt earthquakes and the remaining were originated by the subduction of the Cocos plate. The geographic areas with the highest seismicity were located near Jaco and the Irazú and Poas volcanoes. The highest Modified Mercalli intensity observed was VI+ caused by the Jaco (6.3 Mw) earthquake.

Keywords: Seismic activity, Modified Mercalli Intensity (MMI), Moment Magnitude (Mw), Subduction, Local Faulting.

RESUMEN: Durante el 2017 la Red Sismológica Nacional (RSN: UCR-ICE) localizó 2755 sismos y reportó 216 eventos sentidos por la población, incluyendo cuatro sismos relevantes con magnitudes (Mw) de entre 5,5 y 6,3. La mayoría (91\%) de los sismos percibidos fueron superficiales $(<30 \mathrm{~km})$ y el $63 \%$ tuvo una magnitud baja $(\mathrm{Mw}<3,9)$. El fallamiento local y el regional provocaron el $62 \%$ de los sismos sentidos, mientras que el resto de los sismos fueron originados por el proceso de subducción de la placa del Coco. Las zonas con la sismicidad más alta fueron Jacó y los volcanes Irazú y Poás. La intensidad Mercalli Modificada máxima observada durante el 2017 fue de VI+ debido al sismo de Jacó (Mw 6,3).

Palabras clave: Actividad sísmica, Escala de Intensidad de Mercalli Modificada (MMI), Magnitud Momento (Mw), Subducción, Fallamiento local.
\end{abstract}




\section{INTRODUCCIÓN}

Costa Rica es un país altamente sísmico, con la influencia de un punto triple en donde la Fosa Mesoamericana, que termina contra la Zona de Fractura de Panamá (ZFP), separa las placas del Coco y Caribe. La placa del Coco se subduce por debajo de la placa Caribe y la microplaca de Panamá a una velocidad promedio de $8,5 \mathrm{~cm} /$ año (DeMets, 2001). La ZFP sirve de límite entre las placas del Coco y Nazca al sur de Costa Rica y Panamá, mientras que el Cinturón Deformado del Centro de Costa Rica (CDCCR, Montero, 2001; Marshall, Fisher \& Gardner, 2000) en conjunto con el Cinturón Deformado del Norte de Panamá (CDNP, Adamek, Frohlich \& Pennington, 1988) conforman el límite entre la placa Caribe y la microplaca de Panamá (Fig. 1).

La Red Sismológica Nacional (RSN: UCRICE) es uno de los organismos oficiales nacionales encargados de investigar los procesos sísmicos y volcánicos para contribuir con una adecuada gestión del territorio, promover la cultura de la prevención de desastres e incidir en el mejoramiento de la calidad de vida de la población. La RSN es un convenio de cooperación científico y técnico que funciona desde 1973 entre la Sección de Sismología, Vulcanología y Exploración Geofísica de la Escuela Centroamericana de Geología de la Universidad de Costa Rica (UCR) y el Área de Amenazas y Auscultación Sísmica y Volcánica del Instituto Costarricense de Electricidad (ICE).

La RSN posee una red de 140 estaciones sismológicas (Fig. 1) las cuales son administradas por la UCR (106 estaciones) y el ICE (34). La red sísmica incluye actualmente 21 estaciones de banda ancha y 119 de periodo corto. En muchos casos se incorporaron lecturas de estaciones administradas por otras redes sismológicas ubicadas en Costa Rica, Panamá y Nicaragua disponibles a través del consorcio Incorporated Research Institutions for Seismology (IRIS). Durante el 2017, la detección y la localización automática de los sismos se realizaron a través de los sistemas SeisComP y Earthworm. La lectura manual de arribos de ondas, la localización y el cálculo de la magnitud momento (Mw) se llevaron a cabo usando el programa Hyp (Lienert \& Havskov, 1995) integrado en la última versión del software sismológico SeisAn (Ottemöller, Voss \& Havskov, 2011). Para la localización de sismos se utilizó un modelo siete capas de velocidades de la onda primaria y una razón de velocidades $(\mathrm{Vp} /$ Vs) de 1,74.

Durante el 2017 la RSN localizó 2755 sismos, cantidad que incluye los eventos ocurridos en Costa Rica (sismos locales), algunos ubicados en América Central (regionales) y otros en zonas ubicadas a más de 10 grados de distancia (telesismos). De los 2261 sismos localizados en territorio costarricense (Fig. 2A), 216 fueron percibidos por la población y entre ellos destacaron cuatro sismos con Mw entre 5,5 y 6,3 (Fig. 2B y 3).

\section{CARACTERÍSTICAS DE LOS SISMOS SENTIDOS}

A continuación se describe brevemente la sismicidad registrada por la RSN durante el 2017, con respecto a su distribución temporal y geográfica, así como su profundidad, magnitud (Mw), origen e intensidades más relevantes.

\section{Distribución temporal}

Marzo y noviembre fueron los meses con la menor y mayor cantidad de sismos sentidos (6 y 41), respectivamente (Fig. 4A). Los meses restantes variaron entre 9 y 26 eventos sentidos (Fig. 4A). Los sismos de mayor Mw dentro del territorio costarricense ocurrieron en abril $(5,8)$, setiembre $(5,6)$ y noviembre $(6,3$ y 5,5$)$. El promedio mensual de sismos sentidos fue de 17,1.

\section{Distribución por magnitud}

El sismo de mayor Mw sentido en Costa Rica durante el 2017 fue de 6,3. Este evento ocurrió 


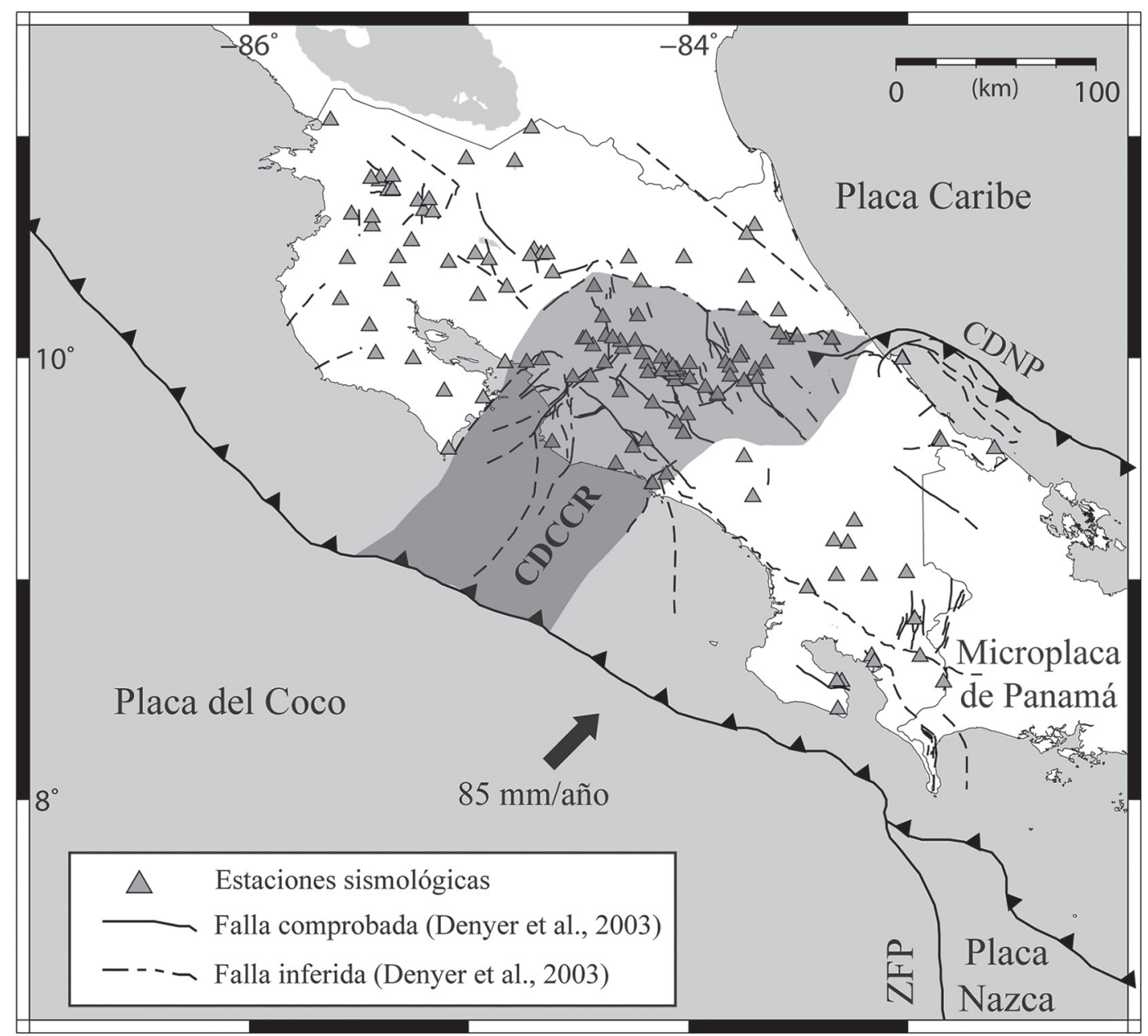

Fig. 1: Contexto tectónico de Costa Rica y estaciones de la Red Sismológica Nacional. CDCCR significa Cinturón Deformado del Centro de Costa Rica, CDNP es el Cinturón Deformado del Norte de Panamá y ZFP, Zona de Fractura de Panamá.

el 12 de noviembre $19 \mathrm{~km}$ al sur de Jacó. Otros tres sismos sentidos tuvieron magnitudes mayores a 5,5. El primero sucedió el 2 de abril, tuvo una Mw 5,8 y su epicentro se localizó al norte del volcán Barú, en Panamá, a $42 \mathrm{~km}$ al este de la frontera con Costa Rica. El segundo ocurrió el 1 de setiembre, tuvo una Mw de 5,6 y su epicentro se ubicó $17 \mathrm{~km}$ al noroeste de Sámara en la península de Nicoya. El tercer evento tuvo lugar el 14 de noviembre, tan sólo dos días después del sismo de Jacó. Tuvo una Mw de 5,5 y se localizó 45 km al oeste de Drake de Osa (Fig. 3).
En total, 12 sismos alcanzaron magnitudes iguales o mayores a 5,0 lo que representa el 5,3 $\%$ de los sismos sentidos del año y el $0,4 \%$ del total de sismos localizados dentro del territorio costarricense (Fig 2B). La mayoría (63\%) de los sismos percibidos tuvieron una $\mathrm{Mw}$ menor a 3,9 (Fig. 4B). Tres sismos sentidos comparten la menor $\mathrm{Mw}(2,3)$ del año y fueron sentidos levemente en las zonas cercanas a los epicentros. El primero sucedió el 30 de marzo $2 \mathrm{~km}$ al sur de Santa Ana en San José; el segundo y el tercer eventos sucedieron el 27 de abril en los alrededores de 


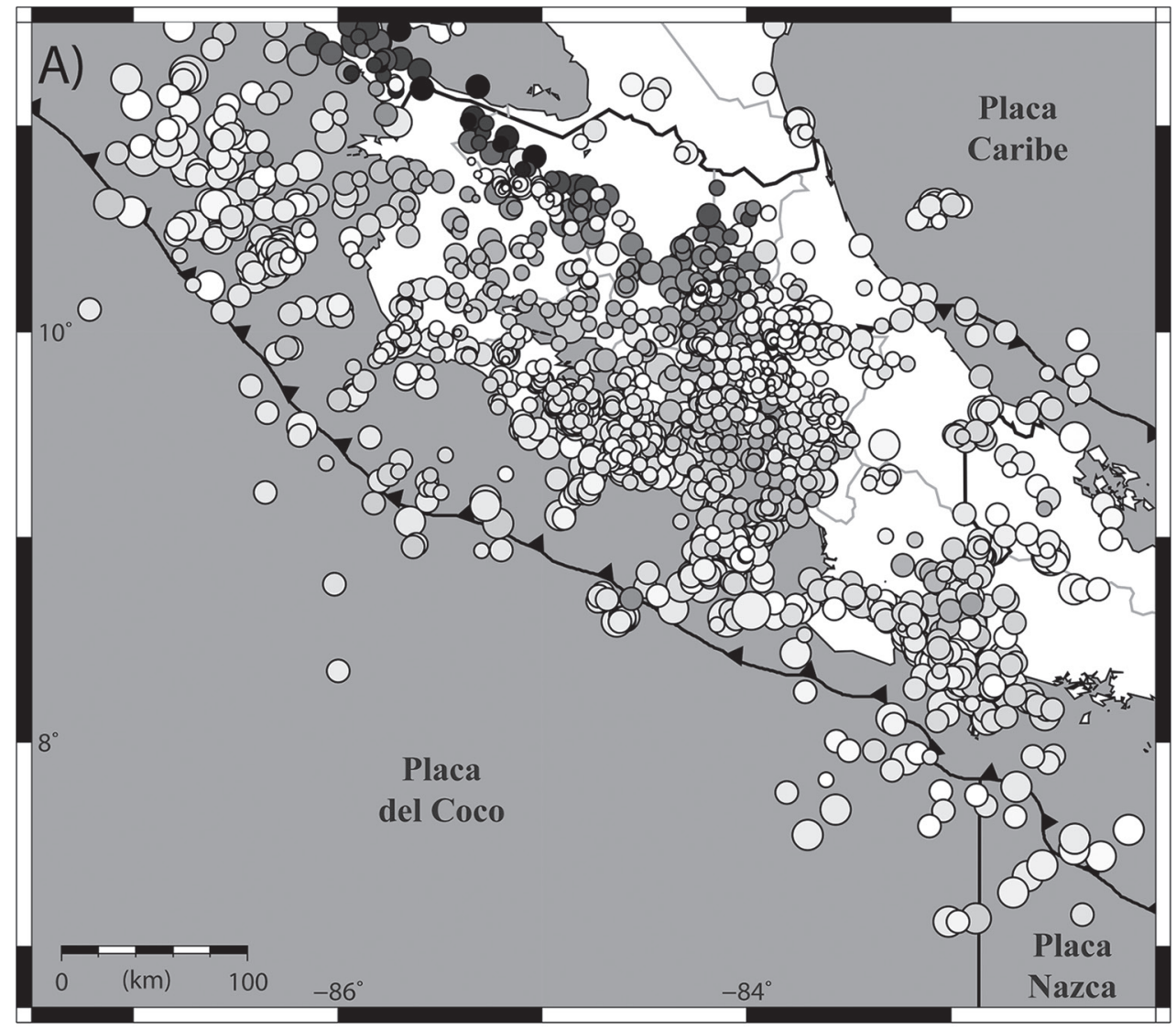

Magnitud (Mw)

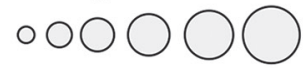

$\begin{array}{llllll}2 & 3 & 4 & 5 & 6 & 7\end{array}$

Profundidad $(\mathrm{km})$

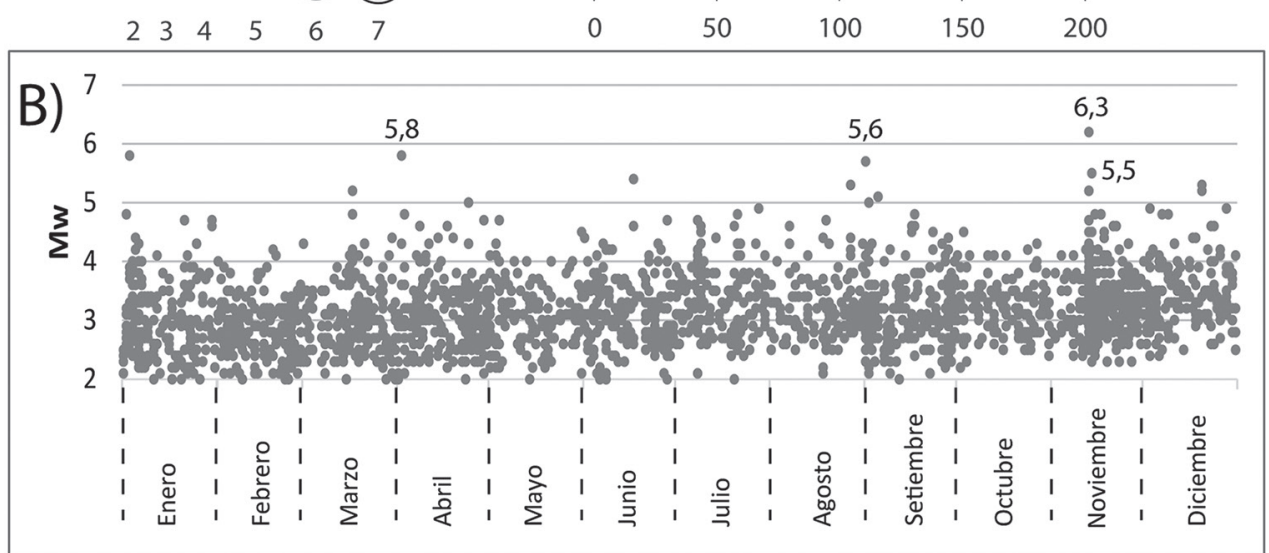

Fig. 2A) Ubicación epicentral y B) Magnitud (Mw) de los sismos localizados por la RSN durante el 2017. 


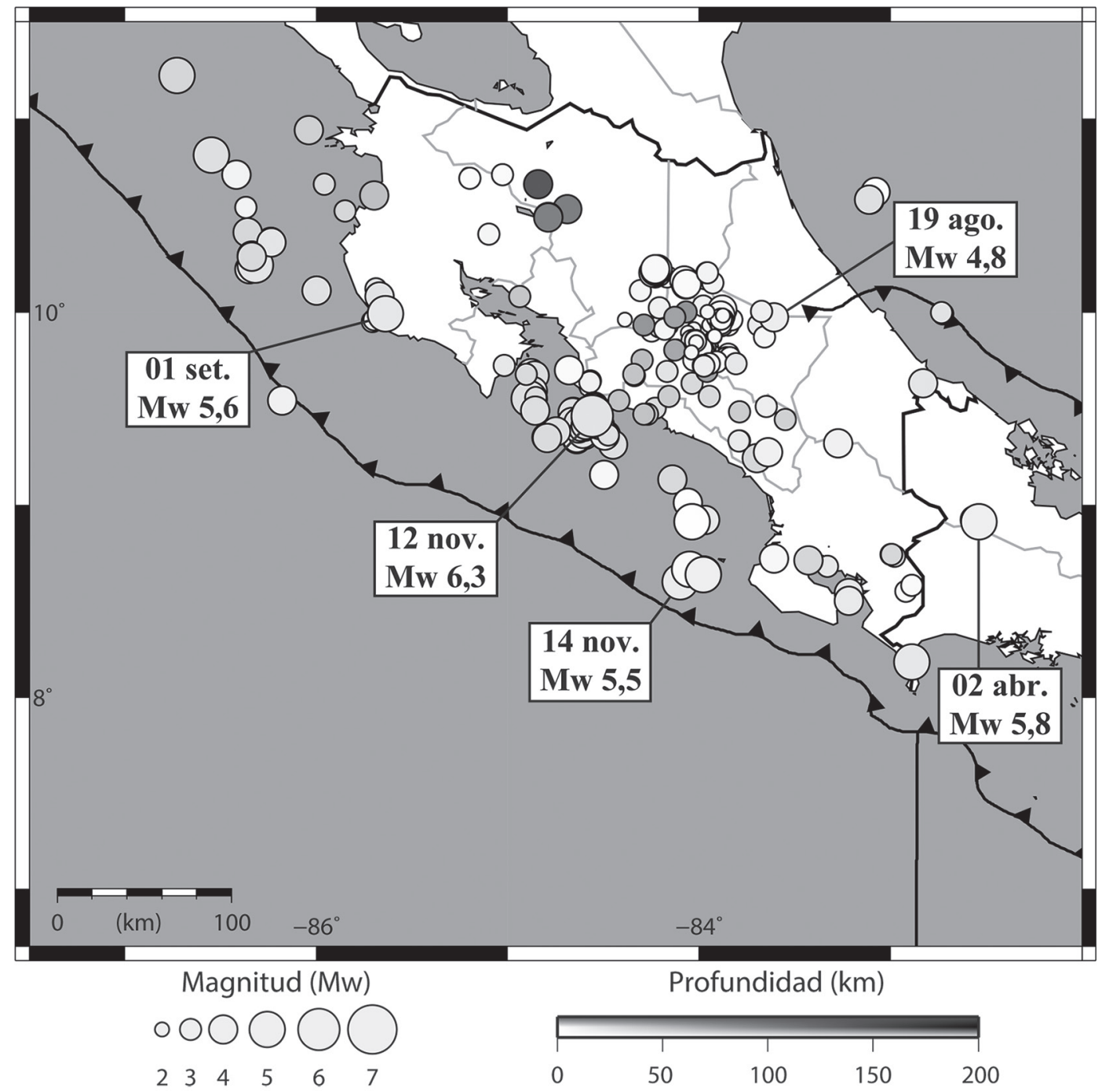

Fig. 3: Ubicación epicentral de los sismos sentidos durante el 2017 estimada por la RSN.

Cartago. La población percibió 27 sismos de baja $\mathrm{Mw}(<2,9$; Fig. 4B) y estos en su mayoría tuvieron epicentros muy cerca de zonas urbanas como Santa Ana, Desamparados y Cartago.

\section{Distribución por profundidad}

El 91\% de los sismos sentidos tuvo profundidades menores a $30 \mathrm{~km}$ (Fig. 4C). De estos even- 

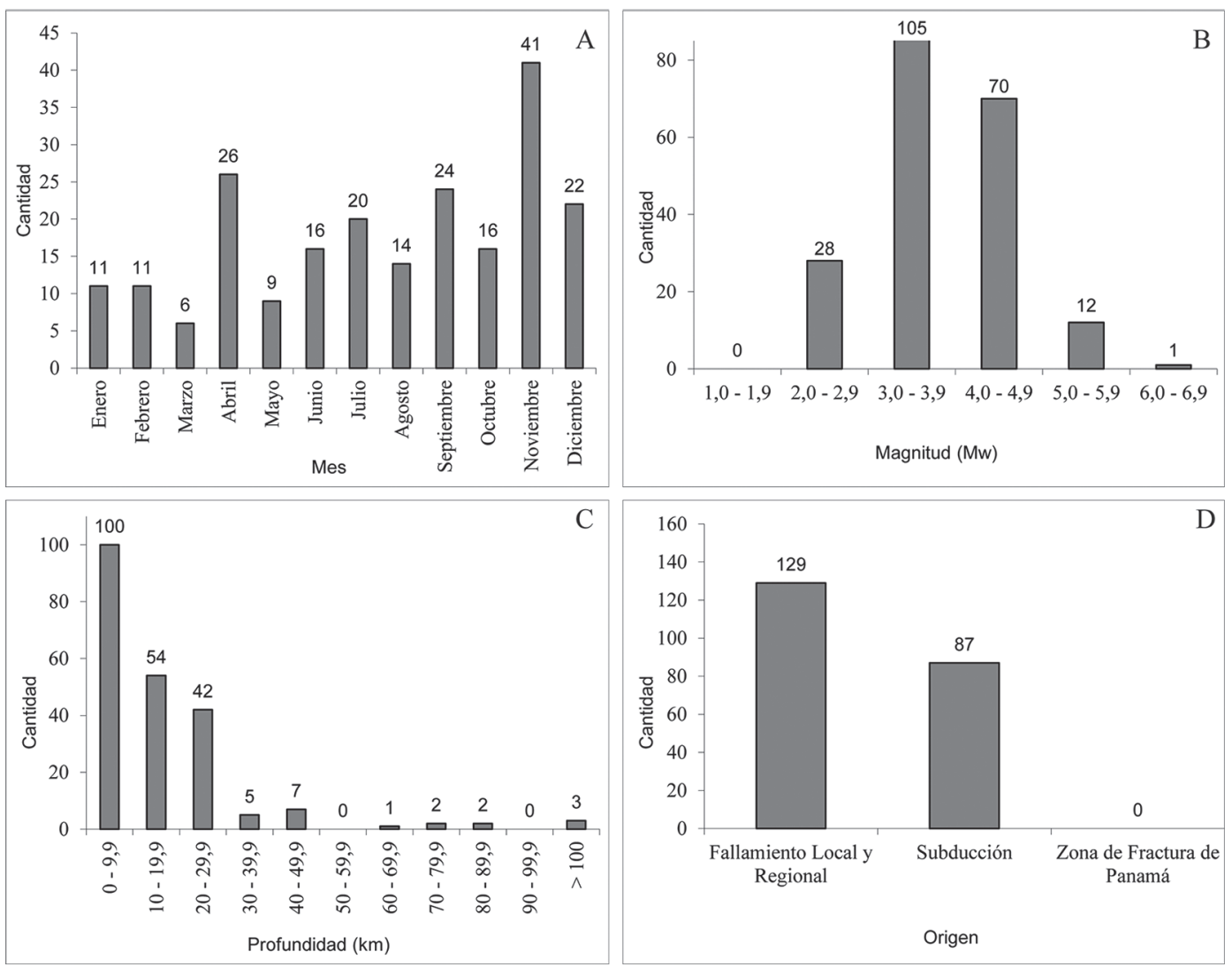

Fig. 4. Distribución de los sismos sentidos en el 2017 por A) mes, B) magnitud, C) profundidad y D) origen. La cantidad de sismos se muestra sobre la barra de cada histograma.

tos, 100 tuvieron hipocentros a profundidades menores a $10 \mathrm{~km}$, mientras que otros 96 tuvieron profundidades entre 10 y $30 \mathrm{~km}$. Solamente 20 sismos sentidos tuvieron su hipocentro a más de 30 $\mathrm{km}$ de profundidad, los cuales se asocian con la deformación interna de la placa del Coco subducida bajo la placa Caribe y la microplaca de Panamá.

Durante el 2017, solamente tres sismos sentidos tuvieron profundidades mayores a $100 \mathrm{~km}$ (Fig. 4C). El primero (105 km) sucedió el 21 de julio, $5 \mathrm{~km}$ al noreste de Tronadora de Tilarán con Mw 4,1; el segundo (102 km) sucedió el 18 de noviembre, localizado $9 \mathrm{~km}$ al sur de Venado en San
Carlos, con Mw 4,5; y el tercero (142 km) sucedió el 28 de diciembre y se localizó $3 \mathrm{~km}$ al suroeste de San Rafael de Guatuso, con una Mw 4,9.

\section{Distribución por origen}

El fallamiento local y el regional originaron el $62 \%$ de los sismos sentidos del 2017 (Fig. 4D). Esto incluye los sismos superficiales $(<30 \mathrm{~km})$ ocurridos dentro de la placa Caribe y la microplaca de Panamá y en las fallas del CDNP y del CDCCR. 


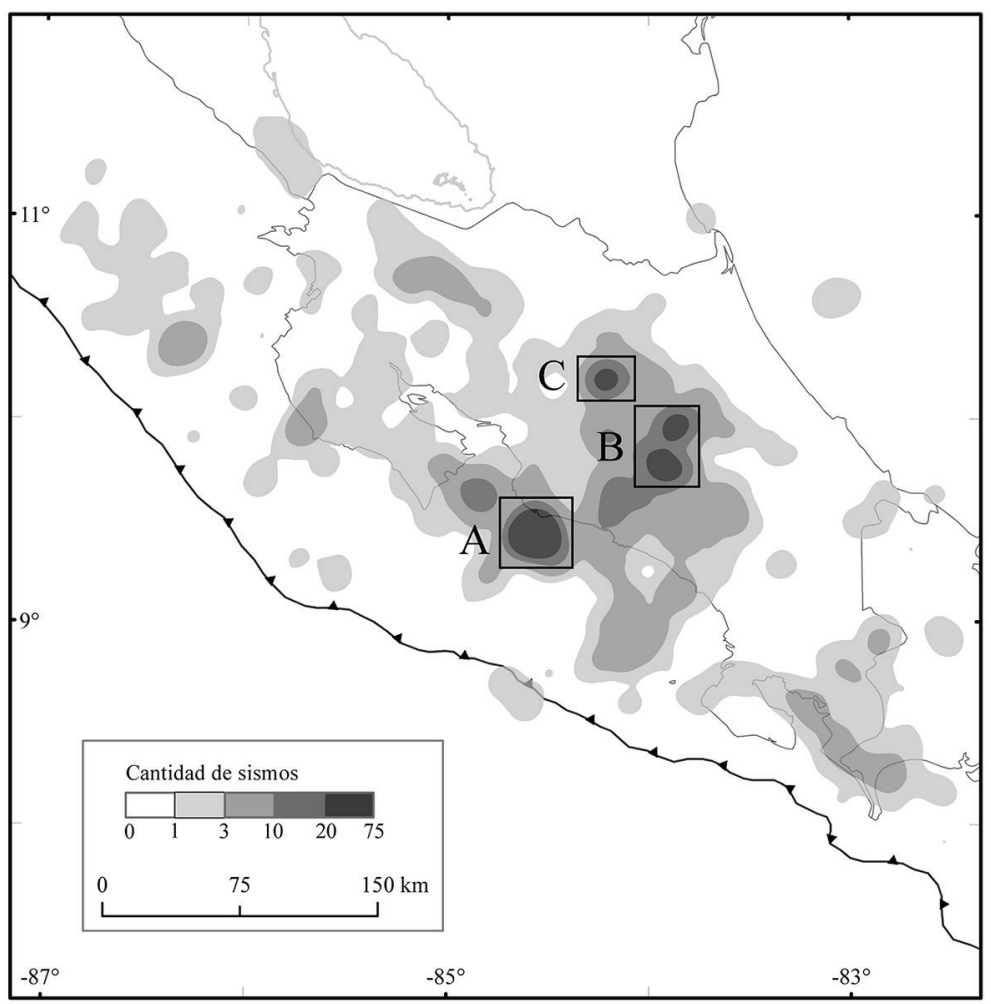

Fig. 5: Distribución espacial de la sismicidad localizada del 2017. Las zonas A, B y C son las que presentaron la mayor cantidad de sismos localizados.

El restante $38 \%$ de los sismos sentidos fueron originados por la subducción de la placa del Coco bajo la placa Caribe y la microplaca de Panamá (Fig. 4D). Este proceso incluye los sismos de la zona sismogénica interplacas y los de profundidad intermedia $(>50 \mathrm{~km})$ que se asocian con la deformación interna y procesos de deshidratación de la placa del Coco que se subduce debajo de Costa Rica. El sismo de Jacó del 12 de noviembre (Mw 6,3) es un ejemplo de los sismos originados en la zona sismogénica interplacas. El mecanismo focal determinado partir de las polaridades de la onda $\mathrm{P}$ y la inversión del tensor de momento indican una solución de fallamiento inverso para este evento (RSN, 2017). Durante el 2017 no se reportaron sismos sentidos asociados con la Zona de Fractura de Panamá.

\section{Distribución geográfica}

Las tres regiones con la sismicidad más alta se ubicaron al sur de Jacó (región A, Fig. 5), en las zonas de Cartago y el volcán Irazú (región B, Fig. 5) y en los alrededores del volcán Poás (región C, Fig. 5). En estas tres regiones ocurrieron entre 20 y 75 sismos durante el 2017. Por otro lado, la Zona Norte y gran parte del Caribe y la cordillera de Talamanca se caracterizaron por una sismicidad muy baja, con menos de tres sismos por cada $100 \mathrm{~km}^{2}$. 


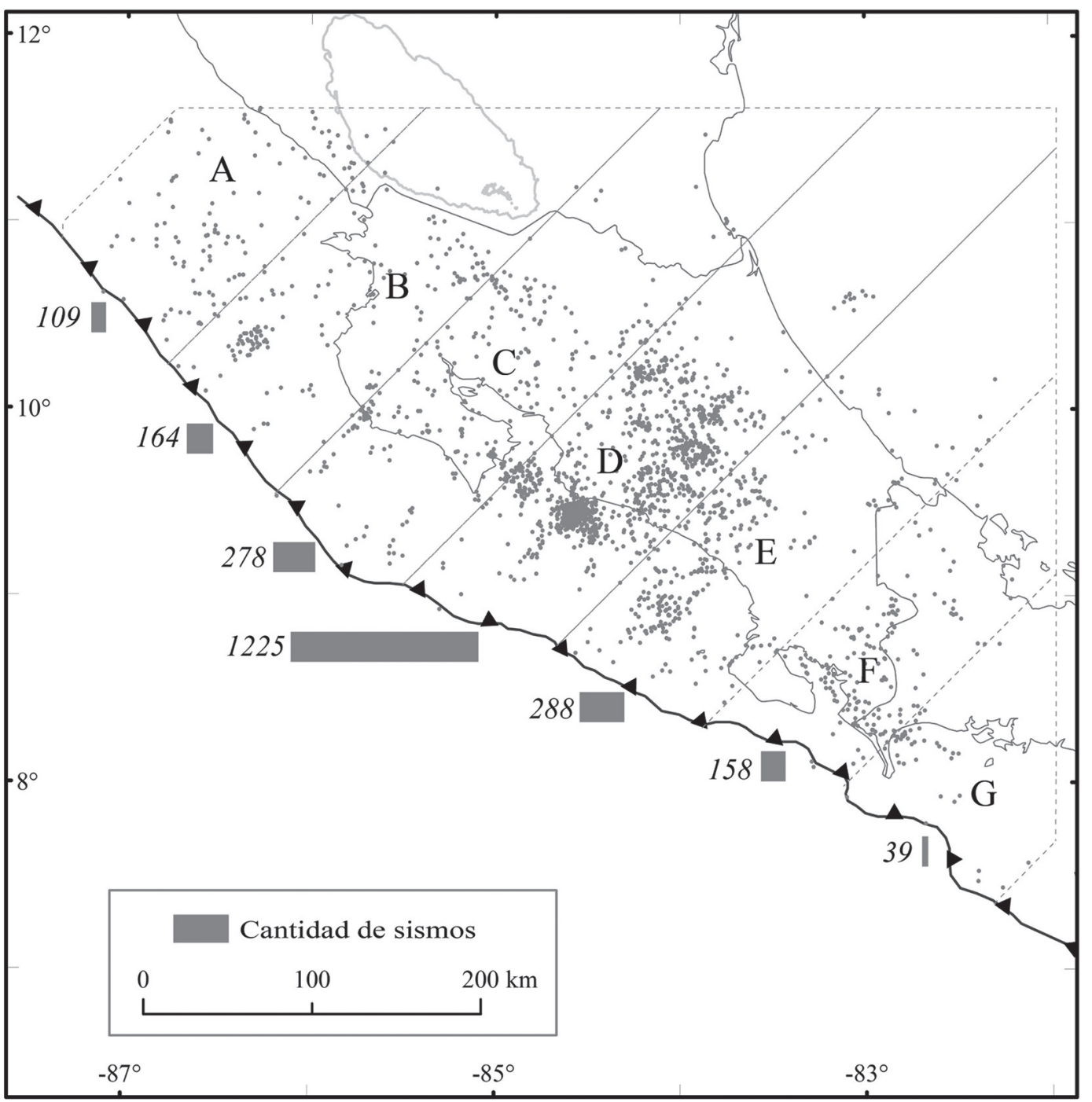

Fig. 6: Cantidad de sismos localizados a lo largo del margen convergente costarricense separado en franjas de $100 \mathrm{~km}$ de ancho.

Los sismos más relevantes en la zona de Jacó (zona A) tuvieron Mw de 6,3 y 5,2 (12 de noviembre) y 4,9 (3 de diciembre). En esta zona, 20 sismos sentidos tuvieron $\mathrm{Mw}>4,0$. En las zonas de los volcanes Irazú y Poás (zonas B y C), los sismos más importantes tuvieron lugar el 27 de setiembre (Mw 4,4) y el 20 de julio (Mw 4,8), respectivamente.
Si se analiza la distribución de la sismicidad a lo largo del margen convergente de la placa del Coco con la placa Caribe y la microplaca de Panamá, es posible observar que la franja más activa comprende la parte central del país, con un total de 1167 sismos, donde ocurrió el sismo de Jacó (Mw 6,3) el 12 de noviembre (región D, Fig. 


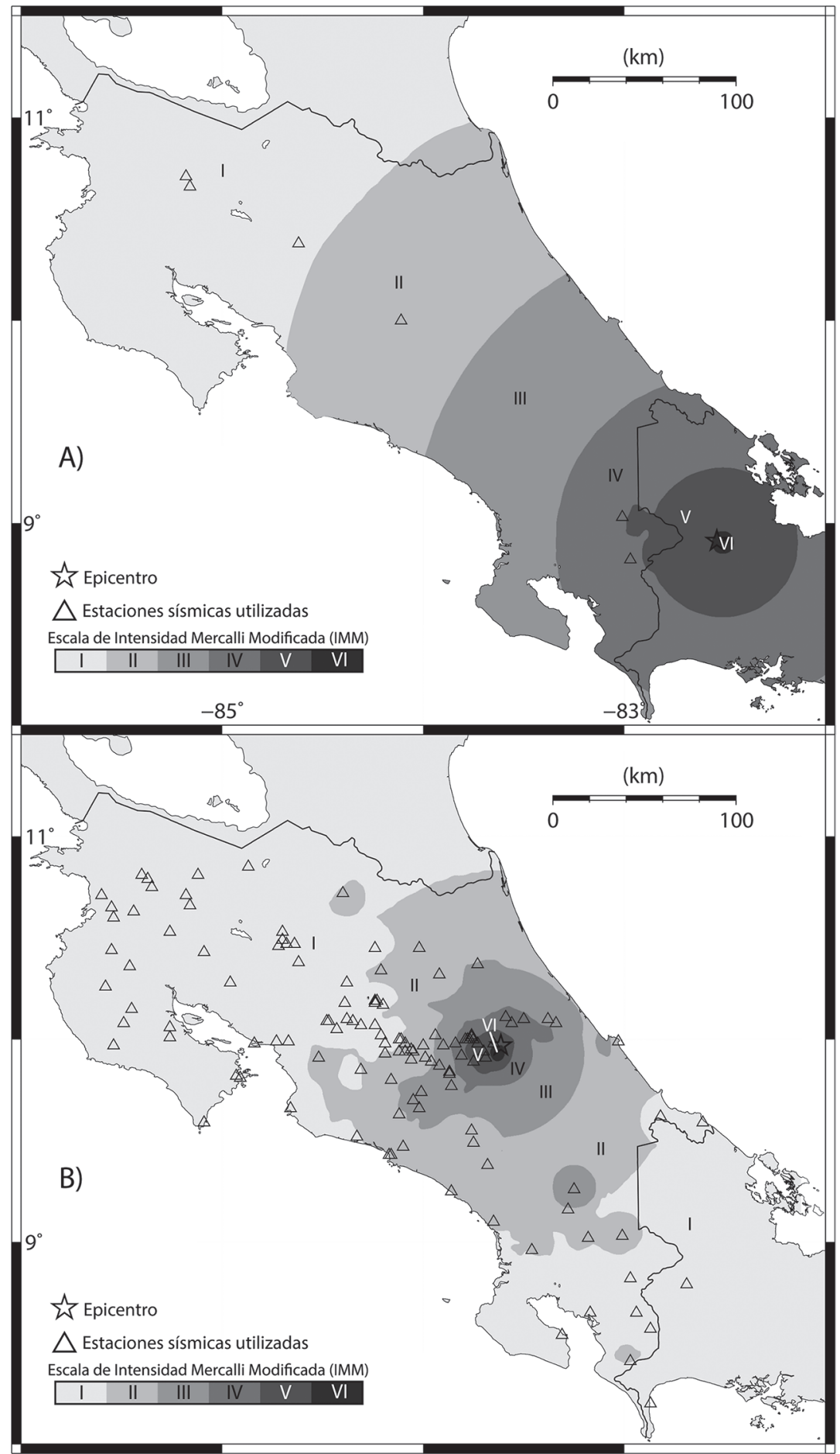

Fig. 7: Intensidades instrumentales para los sismos más relevantes originados por el fallamiento local. A) Sismo del 2 de abril (Mw 5,8). B) sismo del 19 de agosto (Mw 4,8). 


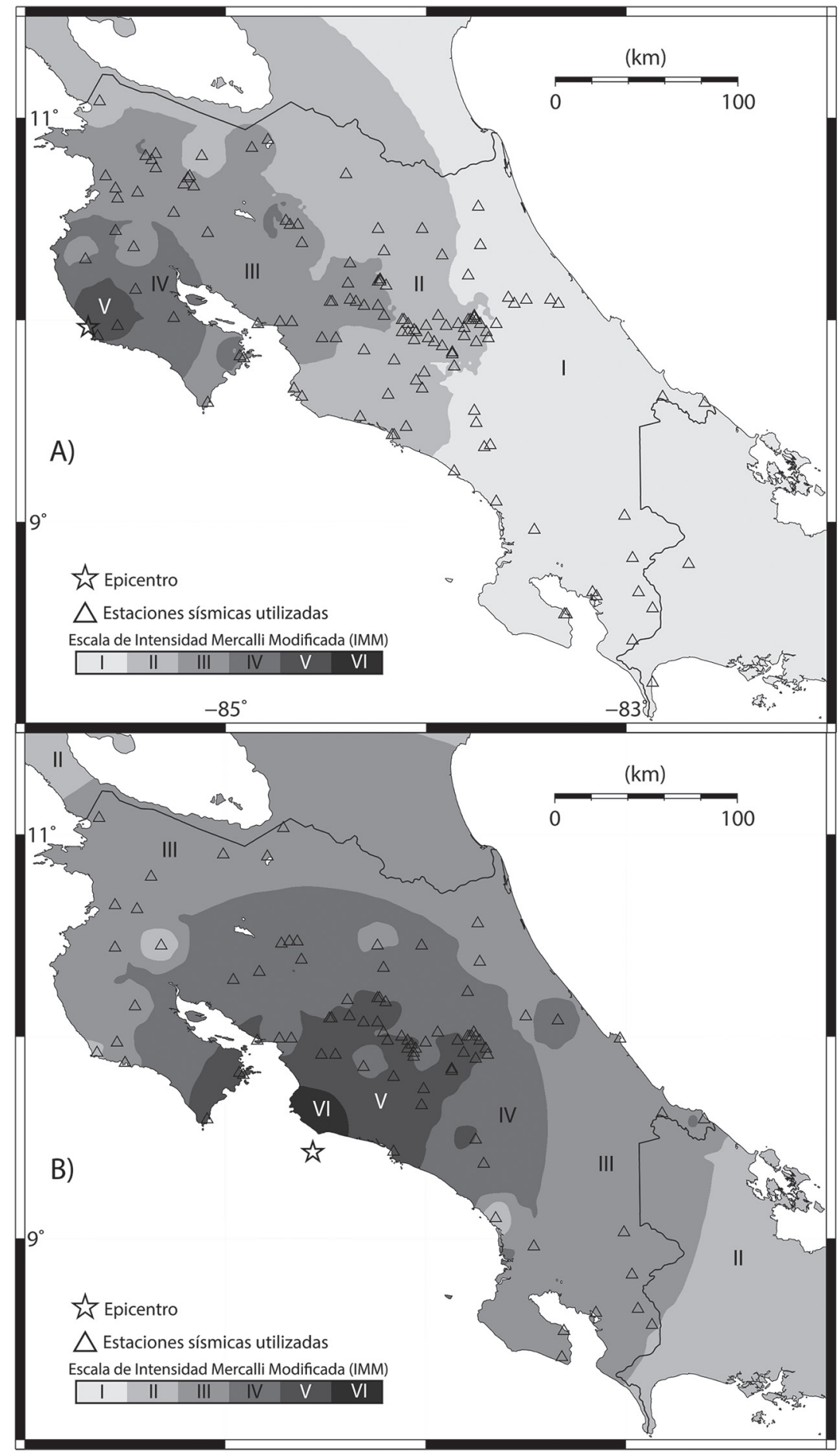

Fig. 8: Intensidades instrumentales para los sismos más relevantes originados por la subducción de la placa del Coco. A) Sismo del $1^{\circ}$ de setiembre (Mw 5,6). B) Sismo de Jacó del 12 de noviembre (Mw 6,3). 
6). En contraste, la franja con menor sismicidad sentida es la región sureste del país, con tan sólo 153 eventos (región F, Fig. 6).

\section{Intensidades}

Durante el 2017 ocurrieron cuatro eventos sísmicos que generaron una intensidad en la escala Mercalli Modificada (IMM) de V a VI+. Estas intensidades fueron determinadas con base en los registros instrumentales de las estaciones sismológicas de la RSN y a través del software ShakeMap (Wald, D. Wald, L. Worden \& Goltz, 2003) que utiliza un modelo de predicción del movimiento de suelo (Zhao et al., 2006) y uno de intensidad (Wald et al., 1999).

El primer evento con intensidades significativas sucedió el 2 de abril (Mw 5,8), localizado $42 \mathrm{~km}$ al este de Sabalito de Coto Brus y a $10 \mathrm{~km}$ de profundidad. Durante este evento se estimaron IMM de hasta VI en la zona epicentral ubicada en Panamá y se observaron intensidades de II y III en San José (Fig. 7A).

El segundo sismo sucedió el 19 de agosto (Mw 4,8), $4 \mathrm{~km}$ al este de Santa Teresita de Turrialba, con hipocentro a $12 \mathrm{~km}$. Para este evento se registró una IMM de VI en la zona del epicentro e intensidades bajas de II en el Área Metropolitana (Fig. 7B). El tercer evento relevante sucedió el $1^{\circ}$ de setiembre (Mw 5,6) en la península de Nicoya, a $16 \mathrm{~km}$ de profundidad. Durante este evento se registró una IMM de $\mathrm{V}$ en la zona epicentral (Fig. 8A) y de II en el Valle Central.

El sismo más importante por las intensidades generadas fue el del 12 de noviembre (Mw 6,3) al sur de Jacó. Este evento fue sentido en todo el país, con IMM de hasta VI+ en la zona epicentral. En Jacó, Esterillos y Parrita se reportó la caída de objetos y daños estructurales leves. En el Área Metropolitana, la IMM fue de IV. Además, fue percibido inclusive en algunas localidades del oeste de Panamá (Fig. 8B).

\section{AGRADECIMIENTOS}

Esta nota técnica es resultado de los proyectos de investigación 113-B5-704, 113-B5-A02 y 113-A1-716 inscritos en la UCR. Agradecemos al personal de la RSN (UCR-ICE) que permite mantener la red de estaciones y los sistemas de localización, especialmente a Luis Fernando Brenes y Jean Paul Calvo. Los asistentes Alejandro Argüello, Allan Sanabria, Roberto Masís y Carolina Fallas realizaron localizaciones de sismos durante el 2017.

\section{REFERENCIAS BIBLIOGRÁFICAS}

Adamek, S., Frohlich, C. y Pennington, W. D. (1988). Seismicity of the Caribbean-Nazca boundary: Constraints on microplate tectonics of the Panama region. Journal of Geophysical Research: Solid Earth, 93(B3), 2053-2075.

DeMets, C. (2001). A new estimate for present-day Cocos-Caribbean plate motion: Implications for slip along the Central American volcanic arc. Geophysical Research Letters, 28(21), 4043-4046.

Lienert, B. R. y Havskov, J. (1995). A computer program for locating earthquakes both locally and globally. Seismological Research Letters, 66(5), 26-36.

Marshall, J. S., Fisher, D. M. y Gardner, T. W. (2000). Central Costa Rica deformed belt: Kinematics of diffuse faulting across the western Panama block. Tectonics, 19(3), 468-492.

Montero, W. (2001). Neotectónica de la región central de Costa Rica: frontera oeste de la microplaca de Panamá. 
Revista Geológica de América Central, 24, 29-56.

Ottemöller, L., Voss, P. y Havskov, J. (2011). Seisan earthquake analysis software for Windows, Solaris, Linux and MacOsX. Bergen, Norway: Dept. Earth Sci., Univ. Bergen.

RSN. (2017). Informe preliminar sismo de Jacó del 12 de noviembre del 2017. Recuperado de http://rsn.ucr.ac.cr/actividad-sismica/reportes-sismicos/9655-informe-preliminarsismo-jaco

Wald, D. J., Quitoriano, V., Heaton, T. H., Kanamori, H., Scrivner, C. W. y Worden, C. B. (1999).
TriNet "ShakeMaps": Rapid generation of peak ground motion and intensity maps for earthquakes in southern California. Earthquake Spectra, 15(3), 537-555.

Wald, D., Wald, L., Worden, B. y Goltz, J. (2003). ShakeMap, a tool for earthquake response. United States Geological Survey Fact Sheet FS 087-03.

Zhao, J. X., Zhang, J., Asano, A., Ohno, Y., Oouchi, T., Takahashi, T. y Fukushima, Y. (2006). Attenuation relations of strong ground motion in Japan using site classification based on predominant period. Bulletin of the Seismological Society of America, 96(3), 898-913. 